\title{
Ocorrência de Psammolestes coreodes Bergroth, 1911 (Hemiptera: Reduviidae: Triatominae) no Estado de Mato Grosso do Sul
}

\author{
Occurrence of Psammolestes coreodes Bergroth, 1911 (Hemiptera: Reduviidae: \\ Triatominae), in the State of Mato Grosso do Sul
}

\author{
Paulo Silva de Almeida ${ }^{1}$, Guilmara Maria do Amaral Gonçalves ${ }^{1}$, Luiz Donizethe Minzão ${ }^{1}$, \\ Ramão Virgilio Genro Larson ${ }^{1}$, Luis Carlos Medina Cristaldo ${ }^{1}$, Walter Ceretti Júnior ${ }^{2}$, \\ Marcos Takashi Obara² e José Maria Soares Barata ${ }^{2}$
}

\begin{abstract}
RESUMO
Neste trabalho, relata-se a ocorrência de Psammolestes coreodes Bergroth, 1911 pela primeira vez no Estado de Mato Grosso do Sul. Em 2006, um espécime foi capturado em um ambiente peridomiciliar do município de Miranda, incluindo o Estado de Mato Grosso do Sul, na distribuição geográfica dessa espécie.
\end{abstract}

Palavras-chaves: Reduviidae. Triatominae. Psammolestes coreodes. Mato Grosso do Sul.

\begin{abstract}
This paper describes the occurrence of Psammolestes coreodes Bergroth, 1911, for the first time in the State of Mato Grosso do Sul. In 2006, a specimen was caught in a peridomestic environment in the municipality of Miranda, thereby including Mato Grosso do Sul within the geographical distribution of this species.
\end{abstract}

Key-words: Reduviidae. Triatominae. Psammolestes coreodes. Mato Grosso do Sul.

0 gênero Psammolestes Bergroth, 1911 compreende apenas de três espécies: Psammolestes arthuri Pinto, 1926; Psammolestes tertius Lent e Jurberg, 1965 e Psammolestes coreodes Bergroth, $1911^{2}$. A distribuição geográfica de Psammolestes arthuri restringe-se, somente a Venezuela. Psammolestes tertius até 0 ano de 2002, em que foi registrada a primeira captura de 26 exemplares desta espécie, em ninhos de garrancheira, também conhecido como graveteiro - Phacellodomus ruber (Passeriformes: Funariidae) - no Município de Porto Rico, Paraná, que faz divisa com Mato Grosso do Sul era registrada apenas nos Estados da Bahia, Ceará, Goiás, Mato Grosso, Minas Gerais, Pará, Paraíba, Pernambuco e São Paulo. Enquanto, Psammolestes coreodes está amplamente distribuída nos países da Argentina (Catamarca, Córdoba, Corrientes, Chaco, Entre Rios, Formosa, Santa Fé, Santiago Del Estero, Salta, Jujuy e Tucumán) Bolívia (Santa Cruz), Paraguai (Central) e Brasil (Mato Grosso), sendo encontrada em habitats

\footnotetext{
1. Laboratório Regional de Entomologia, Núcleo Regional de Saúde, Secretaria de Estado de Saúde, Dourados, MS. 2. Departamento de Epidemiologia, Faculdade de Saúde Pública, Universidade de São Paulo, São Paulo, SP.

Endereço para correspondência: Dr. Paulo Silva de Almeida. Laboratório Regional de Saúde/NRS/SES. Rua Hilda Bergo Duarte 940, Centro, 79806-020 Dourados, MS. Tel: 5567 3421-4672; Fax: 5567 3421-4111

e-mail: psilvadealmeida@yahoo.com.br

Recebido para publicação em 11/11/2008

Aceito em 14/08/2009
}

silvestres associados com ninhos Phacellodomus rufifrons, Phacellodomus sibilatrix, Psedoseisura lophotes, Myopsytta monacha e ninhos de periquitos, ocasionalmente aos arredores de casas e cascas de árvores ${ }^{1245}$.

Psammolestes coreodes embora possa ser infectado experimentalmente, nunca foi encontrado apresentado infecção natural por Trypanosoma cruzi (Chagas, 1909) . $^{3}$

Neste trabalho, é relatada a primeira ocorrência de Psammolestes coreodes Bergroth, 1911 no Estado de Mato Grosso do Sul. Em 2006, o espécime macho estava vivo e foi capturado através de denúncia de moradores no galinheiro (peridomicílio) na Chácara das Piuvas, município de Miranda, a $\left(20^{\circ} 14^{\prime} 26^{\prime \prime}\right.$ ' e $56^{\circ} 22^{\prime} 42^{\prime \prime}$ W) , no Estado de Mato Grosso do Sul. Foi observada a presença de um ninho de Furnariidae próximo do galinheiro que dista aproximadamente 50 metros da casa (Figura 1). 0 exemplar foi identificado pelo o Núcleo Estadual de Entomologia da Coordenadoria de Controle de Vetores/SES de Campo Grande e confirmado após a verificação da genitália em lâmina, pelos especialistas do Laboratório de Entomologia em Saúde Pública do Departamento de Epidemiologia da Faculdade de Saúde Pública (USP), São Paulo, sendo registrado sob o Registro Geral de Triatomíneos n ${ }^{\circ}$ RGT 1682 e devendo ser depositado na Coleção Entomológica do departamento em epígrafe.

A presente nota inclui o Estado de Mato Grosso do Sul, Brasil na distribuição geográfica dessa espécie. 


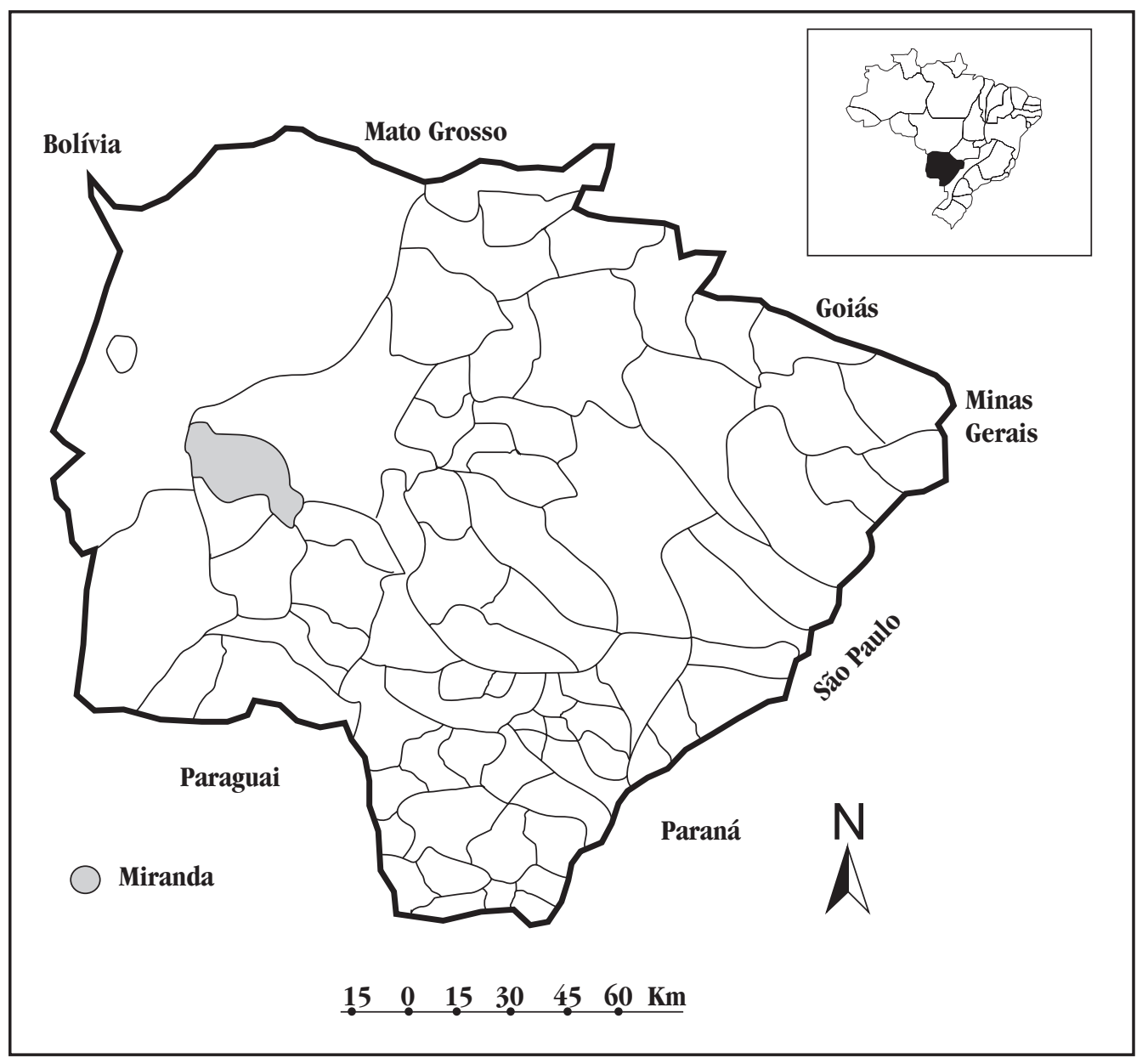

FIGURA 1

Município de Miranda onde foi encontrado o exemplar Psammolestes coreodes no Estado de Mato Grosso do Sul.

\section{REFERÊNCIAS}

1. Carcavallo RU, Girón IG, Jurberg J, Lent H. Bibliographic checklist of the American Triatominae (Hemiptera: Reduviidae). In: Carcavallo RU, Girón IG, Jurberg J, Lent H (eds) Atlas dos vetores da Doença de Chagas nas Américas. Fundação Oswaldo Cruz, Rio de Janeiro, volume I, capítulo 4, p.10-393, 1997.

2. Galvão C, Carcavallo RU, Rocha DS, Jurberg J. A checklist of the current valid species of the subfamily Triatominae Jeannel, 1919 (Hemiptera, Reduviidae) and their geographical distribution, with nomenclatural and taxonomic notes. Zootaxa 202:1-36, 2003. Disponível em www.mapress.com/zootaxa, 2003.
3. Lent H, Wygodzinsky P. Revision of the Triatominae (Hemiptera, Reduviidae) and their significance as vectors of Chagas' disease. Bulletin American Museum Natural History 163: 123-520, 1979.

4. Silva AM, Cristóvão EC, Silva VO, Filho JL, Santos JP, Silva JL, Batata JMS. Primeira ocorrência de Psammolestes tertius Lent \& Jurberg, 1965 (Hemiptera: Reduviidae: Triatominae) no Estado do Paraná, Brasil. In: Resumos do XX Congresso Brasileiro de Entomologia, Gramado, RS, p.10, 2004 .

5. Silveira AC, Feitosa VR, Borges R. Distribuição de triatomíneos capturados no ambiente domiciliar, no período de 1975/83, Brasil. Revista Brasileira de Malariologia de Doenças Tropicais 36:15-312, 1984. 